\title{
A Study of Physiological Signals-based Emotion Recognition Systems
}

Heng Yu Ping, Lili Nurliyana Abdullah, Alfian Abdul Halin, Puteri Suhaiza Sulaiman

Department of Multimedia

Faculty of Computer Science and Information Technology

Universiti Putra Malaysia

43400 UPM Serdang

Selangor Darul Ehsan, Malaysia.

\{ yuping; liyana; alfian; psuhaiza @@upm.edu.my

\section{ABSTRACT}

The use of physiological signals is relatively recent development in human emotion recognition. Interest in this field has been motivated by the unbiased nature of such signals, which are generated autonomously from the central nervous system. Generally, these signals can be collected from the cardiovascular system, respiratory system, electrodermal activities, muscular system and brain activities. This paper presents an overview of emotion recognition using physiological signals. The main components of a physiological signals-based emotion recognition system are explained, including discussion regarding the concepts and problems about the various stages involved in its framework.

\section{Indexing terms/Keywords}

Classification; Emotion Recognition; Feature Extraction; Physiological Signals

\section{Academic Discipline And Sub-Disciplines}

Computer Science

\section{SUBJECT CLASSIFICATION}

Physiological Signals Processing

\section{Council for Innovative Research}

Peer Review Research Publishing System

Journal: INTERNATIONAL JOURNAL OF COMPUTER AND TECHNOLOGY

Vol 11, No. 1

editor@cirworld.com

www.cirworld.com, member.cirworld.com 


\section{INTRODUCTION}

Emotion is dynamic and and highly subjective. It arises spontaneously with short-lived affective states and is often accompanied by physiological changes in evoking human reactions and expressions. Emotion is also important to convey how one actually feels as it is sometimes difficult to do so using the limited set of verbal resources alone. Lately, including emotion for the enrichment of user-computer experience has become a focus in the area of Human Computer Interaction $(\mathrm{HCl})$.

Emotion oriented computing is intrinsically complex as it consists of multiple components that represent different aspects of emotions. These components are cognitive appraisal, action tendencies, motor expression, physiological symptoms and subjective feelings [1] [2]. All of these components need to be interdependent and concurrent in order to evoke the emotions [3].

Emotion recognition through facial expressions, speech recognition and gesture movements have been proposed over the last decade where satisfactory results have been reported for specific applications [4]. However, the performances for the approaches such as facial expression recognition system are relying on the performance capture [5]. Besides that, such behavioral modalities might be easily triggered by intentional control or human social masking. For example, anger can masked by the happy face of a person possessing high emotional quotient (EQ). Thus, to obtain a promise result in emotion recognition, an insight into human feelings has to be considered [6]. In affective computing, physiological signals have become a robust emotional channel to combat the artifacts created by human social masking [4].

This paper aims to study the methods used in recognizing emotion using physiological signals. The rest of the paper is structured as follows. Section II discusses the widely used emotional models, followed by an evaluation of the commonly utilized emotion recognition physiological measures in Section III. Section IV outlines the general framework for physiological signals-based emotion recognition systems. Finally, Section V concludes the paper.

\section{EMOTION REPRESENTATION}

Due to individual differences, various ways are used to express certain emotions. Generally, emotion can be represented using the discrete approach by Ekman [7], or the dimensional approach by Lang [8].

The discrete approach claims that the core emotions, which include happiness, sadness, surprise, anger, disgust fear, curiousity and acceptance, evolved from other emotions. For example, disappointment is derived from sadness and surprise [9].

The dimensional approach is derived from cognitive theories, which map the emotions into a two-dimensional bipolar model of arousal and valence [10]. Valence, which ranges from negative to positive, is used to represent the pleasantness of the stimuli. Arousal, on the other hand is the activation level that ranges from calm to excited [4]. For example, sadness has negative valence and low arousal, whereas anger has negative valence but high arousal.

Both the discrete and dimensional approaches are widely used due to their simplicity and integrability. The different emotional labels can be plotted by mapping the discrete core emotions in the dimensional approach as shown in Figure 1.

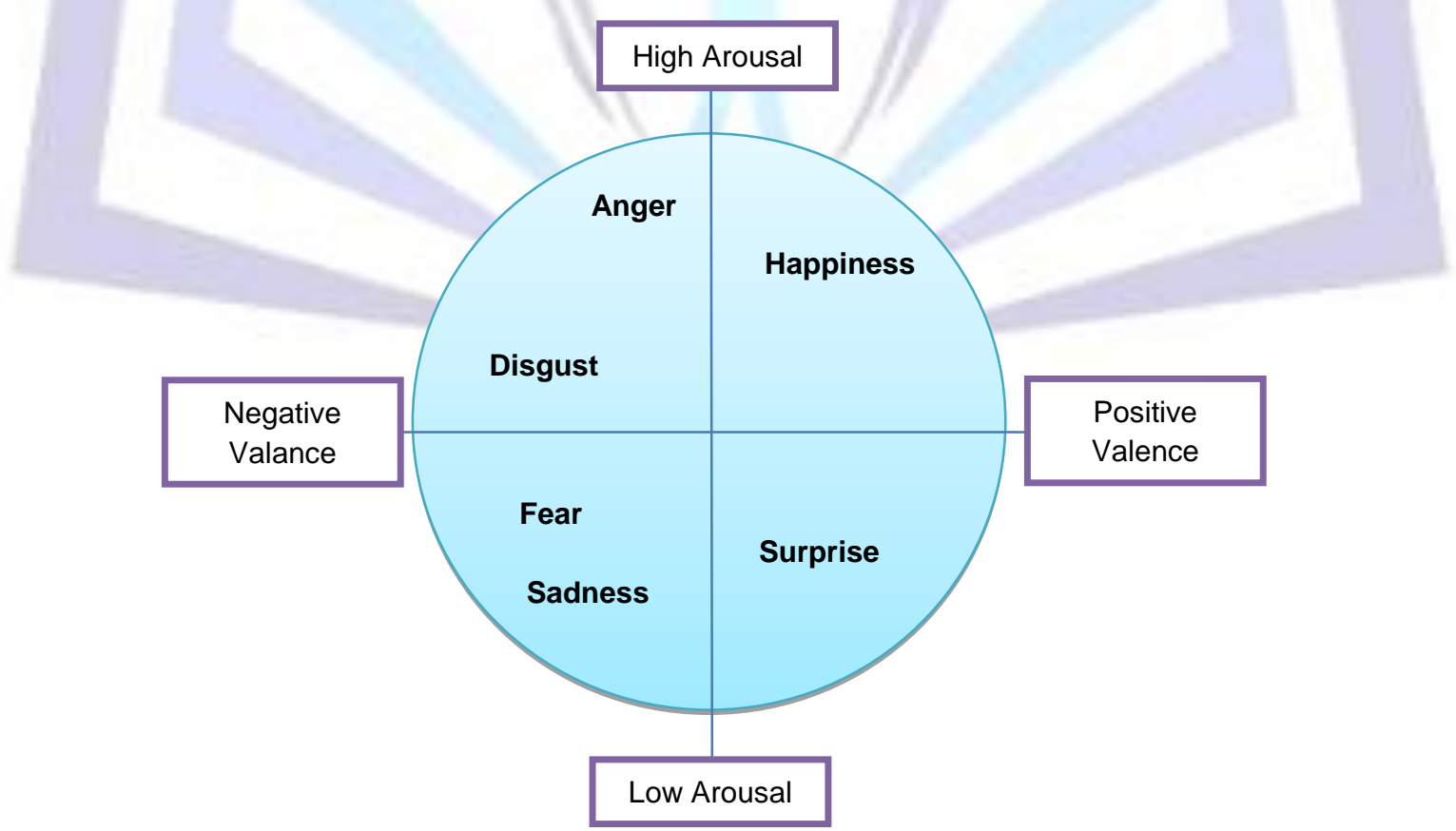

Figure 1: Discrete Arousal-Valence Model 


\section{PHYSIOLOGICAL MEASURES IN EMOTION RECOGNITION}

Physiological signals are originating from autonomic and central nervous system. According to [6] and [11], five physiological measures are commonly adopted in $\mathrm{HCl}$ : cardiovascular system, electrodermal activity, respiratory system, muscular system and brain activity.

\section{Cardiovascular System}

The human cardiovascular system is a closed circulatory system powered by the heart, where the left and right ventricles provide adequate oxygenated blood to the body. Electrocardiogram (ECG) is used to measure the electrical activity associated with the muscular contraction and relaxation of the heart by placing an array of electrodes on the body's surface.

Generally, the ECG traces the sequence of depolarization and repolarization of the atria and the ventricles of the heart. The ECG signal is composed of the P wave, the QRS Complex (ventricular depolarization) and the $T$ wave (ventricular repolarization), as shown in Figure 2. The readings from the ECG wave is then utilized to derive the heart rate (HR), heart rate variability (HRV), blood volume pressure (BVP) and other measurements. The HR can be obtained by measuring the time duration between successive $R$ waves. On the other hand, the BVP can be calculated from the HR and HRV measurements.

In emotion recognition, ECG can be reliably used to classify negative and positive emotions such as stress and happiness [4]. Besides that, ECG signals can be obtained relatively easily through wearable sensors.

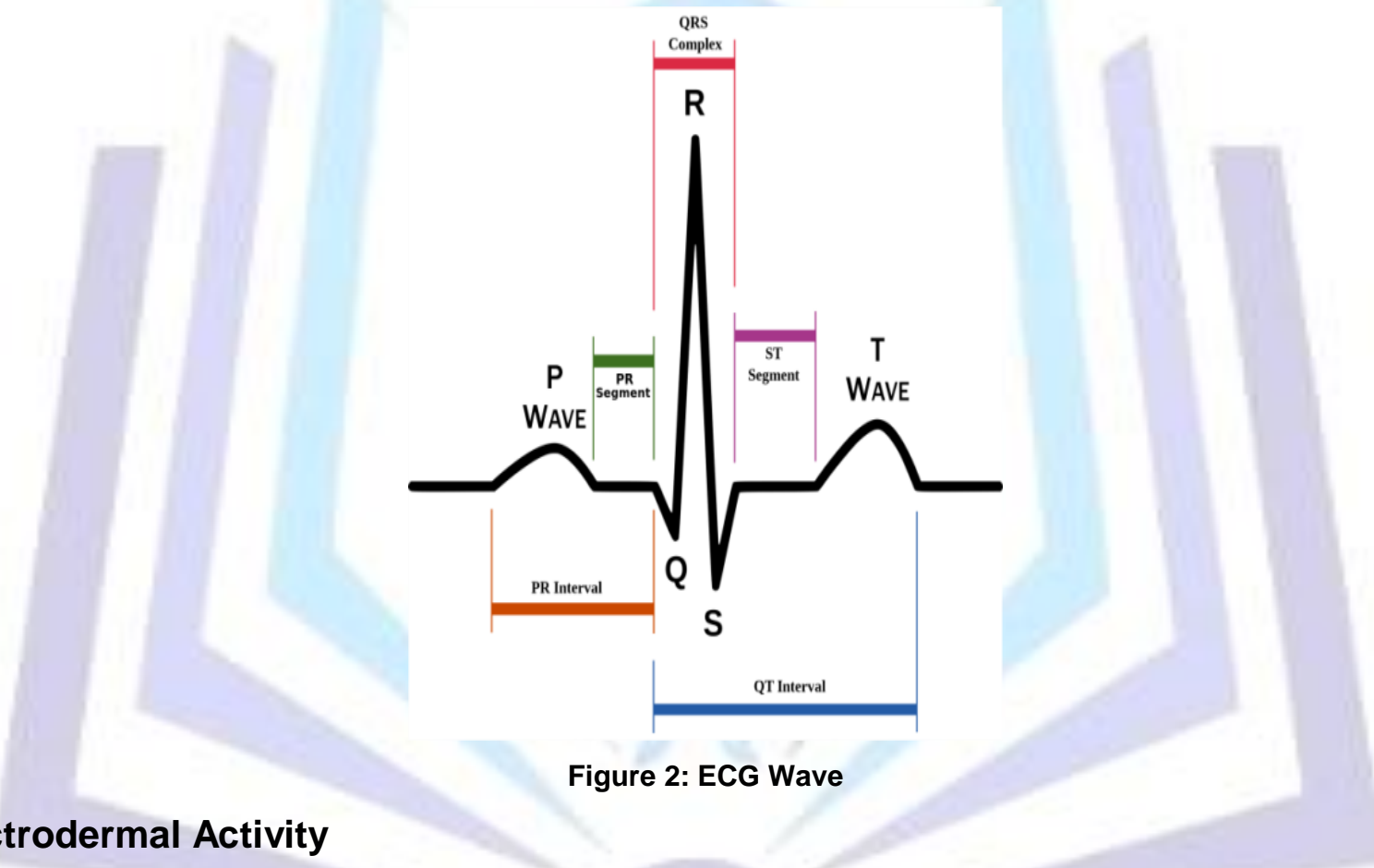

Electrodermal activity (EDA) or sometimes referred to as Galvanic skin response (GSR) is another indicator that prompts emotion to reflect the sympathetic nervous activity. In the past, many studies have shown that EDA signals have good potential in exploring emotions such as attention, arousal difference, anxiety and excitement [12].

EDA describes the ability of the human skin in handling electricity [13]. EDA sensors are usually placed on the fingers to measure the skin's current conduction or resistance property after applying a fixed small voltage to the skin. Electrical changes in the skin are caused by the activity of the sweat glands, which are present throughout the human body. When there is an increase in arousal or cognitive workload, the sweat gland activity increases, thereby increasing the level of skin conductance.

EDA signals are one of the most robust physiological signals in emotion recognition as they indicate sympathetic-centered response whereby the emotion is easily evoked during stimuli presentation [12]. However, EDA signal measurement may be confounded by environmental conditions, different types of physical activities and placement of the sensors. Although controlled experimental conditions can be applied, there is still room for improvement to reduce these artifacts.

\section{Respiratory System}

Respiration (RSP), another form of autonomic control, may change in response to the increase in heart rate and sweating activity [13]. RSP measurements refer to the respiration rate (RF) and the relative breath amplitude (RA) or depth of the breath. 
Generally, the respiratory measures can be recorded by wearing elastic stretch sensors placed on the upper chest and the lower abdomen. The amount of the stretch (chest expansion) is recorded and is used for RF and RA calculations. Previous studies in [4][13][14] have shown that RSP patterns highly rely on an individual's emotional state changes. RF generally decreases during relaxation and bliss, but increase during anger and anxiety. Besides that, irregular RSP patterns are detectable when dealing with negative valence. To obtain a complimentary result in emotion recognition, comprehensive assessment of ECG, EDA and RSP is suggested.

\section{Muscular System}

Muscle activity is a common observation used to recognize the correlation between emotion and physiological signals [4]. An electrical current is transmitted from motor neurons to drive muscles to contract and relax; and these activities are captured using an Electromyogram (EMG) [15]. On one hand, EMG is used to capture the electrical activity of muscles. On the other hand, EMG is used to measure the conducting function of the nerves.

In general, surface EMG is more practical compared to intramuscular EMG to collect signals for emotion recognition. Surface EMG signals can be collected by placing the sensors on the surface of the face, arm or leg, while intramuscular EMG involves inserting a needle electrode into the muscle. The frequency (speed) and amplitude (strength) of the surface EMG signals travelling between the sensor points are then measured. In most cases, the amplitude changes in EMG signals are directly proportional to muscle activity

\section{Brain Activity}

Brain is the center of the nervous system responsible to coordinate the activity of the peripheral nervous system [14]. The electroencephalogram (EEG) is used to measure the brain's spontaneous electrical activity along the surface of the scalp.

Today, EEG-based emotion recognition has received significant attention in $\mathrm{HCl}$, which is partly due to the development of wearable EEG sensors. EEG signals data collection process is faster, easier and less invasive compared to other methods such as via functional Magnetic Resonance Imaging (FMRI) [16].

The performance of EEG-based emotion recognition relatively depends on the spatial location, response time and frequency structure of the brain waves [14]. Besides that, EEG signals usually require more than 3 electrodes consists of Fp1, Fp2 and Fpz as illustrated in Figure 3, mounted on the forehead to collect the measurements. This setup can be cumbersome for feature extraction and also prone to noise, compared to other physiological signals with less electrodes.

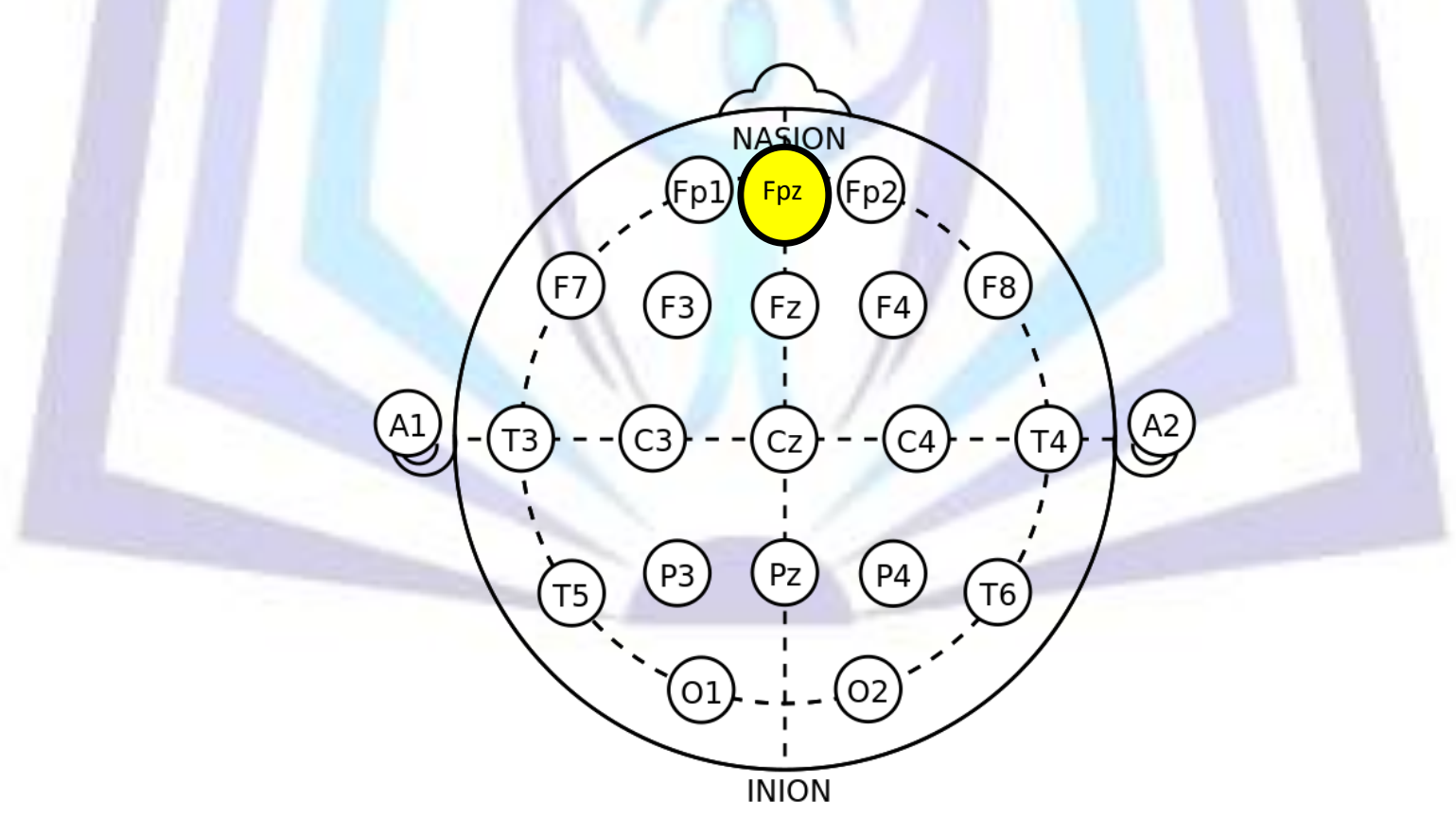

Figure 3: 10-20 system of electrode placement

\section{PHYSIOLOGICAL SIGNAL BASED EMOTION RECOGNITION FRAMEWORK}

Different physiological measures are used for different states of emotions. To recognize happiness for instance, ECG and RESP are adequate measurements to attain high accuracy, whereas EDA alone is sufficient to recognize anxiety. Generally, the processing steps involved to recognize emotions are similar regardless of the different types of physiological signals used. 
The overall framework for a physiological signals-based emotion recognition is illustrated in Figure 4. Brief descriptions for each stage are presented below.

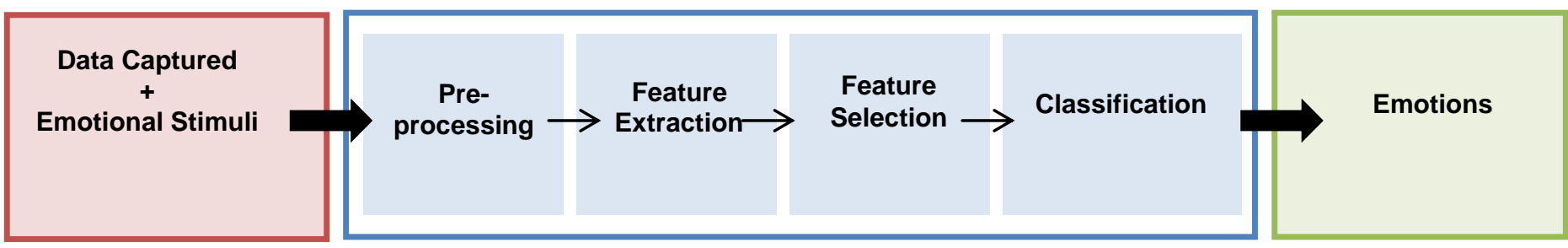

Figure 4: Overview of the Physiological Signals based Emotion Recognition Framework

\section{Data Acquisition - Emotional Stimuli}

Emotional stimuli are important to evoke the respective emotion during the data acquisition stage. There are generally two ways to evoke emotions: personal imagery from previous experiences or audio-visual material shown to the participants [17]. Most of the experiments prefer to use audio visual stimuli because the experiences from memory are difficult to spontaneously call up to evoke the targeted emotion.

International Affective Digitized Sounds (IADS)[18] and International Affective Picture System (IAPS)[19] are two non-profit libraries providing emotional annotated sounds and images for research purposes. The study done by [20] claims that films achieve better results in evoking the target emotions. However, the same stimuli might not evoke the same emotional states to all participants [21]. A funeral scene may easily evoke sadness for a female, but not for a male. A comedy may evoke happiness but not excitement for an adult. Thus, selecting well suited stimuli is important to gather the quality data.

\section{Preprocessing}

Readings from sensors might be inaccurate due to body movement during data acquisition. To avoid such noises and artifacts, a preprocessing stage is needed. Some technique can be used such as a low pass and smoothing filter. These filters may vary for different types of physiological measurements. ECG and facial EMG signals best adapt to the low pass filter at $100 \mathrm{~Hz}$ and $500 \mathrm{~Hz}$. The moving average filter is used to preprocess RSP and EDA signals [22]. During EMG signal collection, the muscle contraction frequency for each sensor point may vary. Thus, extra preprocessing is required for EMG signals. In [4], an adaptive bandpass filter is used to remove the noise and artifacts from the EMG signal generated by the heartbeat and RSP.

Compared to other physiological measurements, EEG signals generate more noise and artifacts due to the large number of electrodes and its sensitivity to the face movement such as eye blinks or eyebrow raises. Bos et al. [17] used a bandpass filter provided by EEGLab for Matlab to remove the noises and artifacts of EEG signals. At first, Fourier frequency analysis [23] is adapted to split up the raw EEG signals. The detected noise is then removed using the bandpass filter. After noise filtering, the targeted frequencies are retransformed to be used for further processing.

Apart from noise filtering, certain physiological signals will be segmented into different samples and normalized by its mean and standard deviation before proceeds to the next stage.

\section{Feature Extraction}

Feature extraction in physiological signals processing involves the extraction of statistical features in the time domain, frequency domain, time-frequency domain and other domains from the preprocessed physiological signals. As the input channels are different for each physiological measurements, feature extraction is generally performed separately. The time domain statistical feature (root-mean-square) is extracted from EMG signals. This feature describes the power of the signal, which can determine the level of strength or fatigue of a muscle. The feature extracted from ECG signals is from the frequency domain, namely the power spectrum that identifies the mental state changes from relaxation to stress.

To transform time domain features to frequency domain features, certain techniques are applied such as wavelet transform (WT), fast Fourier transform (FFT), Hilbert Huang transform (HHT) and principal component analysis (PCA). However, physiological signals are non-stationary (the signals hardly remain constant over time). Thus, it is crucial to select the best suited technique for feature extraction. Compared to the other previously mentioned techniques, the wavelet transform is particularly useful in extracting features from aperiodic and non-stationary signals [24].

S.Koelstra et al. [25] extracted a total of 106 features: time frequency, zero crossing rate, silence and others from six physiological signals namely, GSR, RSP, EMG, blood volume pressure(BVP), skin temperature(SKT) and EEG . On the other hand, Zong and Chetouani [26] extracted 28 features by using the proposed fission and fusion approaches based on 
the HHT from four physiological signals (ECG, RSP, EMG and GSR) to recognize joy, anger, sadness and pleasure. C.Maaoui et al. [27] acquired 30 features from each five physiological signals (BVP, EMG, SC, SKT and RSP) using six different affective states (amusement, contentment, disgust, fear, neutrality and sadness) by using the IAPS as stimuli.

From this information, it is noted that the number of features extracted depends on the type of physiological signal. For example, more features are required when dealing with EEG (Table 1). In addition, the number of emotions to be recognized will affect the number of extracted features. For instance, 28 features may perform well to recognize four different emotional states, but might not work well for six emotional states.

Table 1: Features extracted from different physiological measures

\begin{tabular}{|l|l|}
\hline Type of Signal & Features \\
\cline { 2 - 3 } & $\begin{array}{l}\text { Mean amplitude rate, Average and Standard Deviation of HR, } \\
\text { Mean of absolute values of first differences, Mean Frequency, } \\
\text { Median Frequency }\end{array}$ \\
\hline ESP & $\begin{array}{l}\text { Mean amplitude rate, Conductance responses, Rate of skin } \\
\text { conductance, Mean of absolute values of first differences, } \\
\text { Mean rise duration of skin }\end{array}$ \\
\hline EMG & $\begin{array}{l}\text { Mean amplitude rate, Respiration rate, Average respiration } \\
\text { signal, Mean of absolute values of first differences, Average } \\
\text { peak to peak time, Median peak to peak time }\end{array}$ \\
\cline { 2 - 3 } EEG & $\begin{array}{l}\text { Mean Value, Root Mean Value, Standard deviation } \\
\text { Fpz alpha band, Fpz beta band, F3/F4 alpha band, Fpz beta } \\
\text { frequency, F3/F4 beta power or power ratio, Fpz alpha and } \\
\text { beta band power, F3/F4 alpha and beta band power }\end{array}$ \\
\hline
\end{tabular}

\section{Feature Selection}

The ultimate goal of feature selection is to select the most relevant features from the extracted features in order to differentiate each emotional state. Removal of redundant and irrelevant features can decrease the computational time and avoid classifier over-fitting.

Several feature selection techniques have been proposed by the research community, such as sequential forward selection (SFS), sequential backward selection (SBS), sequential floating forward selection (SFFS), margin based feature selection and Fischer projection. Among these, SFS and SBS are frequently adopted in emotion recognition. SFS starts with an empty set where the best fit feature is inserted in every step. In contrast to SFS, SBS reduces the worst features from a full set of features [28]. J. Kim et al. [4] used SBS and pseudo inverse linear discriminant analysis (pLDA) classifier to obtain average recognition accuracy ranging from 87 to 98 percent for arousal, valence and four emotional classes.

In order to obtain promising results, the criterion to employ the appropriate feature selection technique can depend on the classifier. Most feature selection techniques run in offline mode. Thus, there is still room for improvement to run feature selection in real-time as the demand for $\mathrm{HCl}$ to imply the real time response increased relatively [6].

\section{Classification}

In the context of this framework, classification involves categorizing the set of feature measurements into its respective emotional state. This is normally done using machine learning techniques such as supervised learning, unsupervised learning and/or semi-supervised learning. In the supervised case, the label for the training data, along with their respective class label, are prepared. The learning process then generates a hypothesis that generalizes future unseen patterns into the supposed class. In contrast to unsupervised learning, input patterns are unlabeled. The algorithm has to seek out similarites between the data to define groups or clusters that a particular pattern might belong to. Semi-supervised learning uses both labeled and unlabeled data for training [6][29].

Recently, various techniques and approaches have been proposed in order to pursue high accuracy in classification. Picard [30] attained 81\% classification accuracy for eight emotional classes from four physiological signals using Maximum a Posteriori (MAP). A hybrid SFFS with Fisher Projection feature selection method is proposed by them to select the relevant features before MAP classification. Arroyo [31] and his team developed a bio-affective computer interface that can recognize four emotional states using three physiological signals. A probabilistic neural network (PNN) was used as the classifier, which managed to obtain a classification accuracy of $84.46 \%$. Jang et al. [32] used a support vector machine (SVM) to classify three different emotions (boredom, pain and surprise).

Several other classifiers were also used in emotion recognition such as AdaBoost (AB), nearest neighbor(NN) and Naive Bayes (NB). Each has its own strength to work with the given data set. The classification accuracy seems to depend on the number of physiological signals being measured. However, it remains a challenge to compare different classification algorithms as the emotion recognition systems are tested on different data sets. 


\section{CONCLUSION}

The implementation of a physiological signals-based emotion recognition system involves several stages: physiological signal data acquisition, preprocessing, feature extraction, feature selection and classification. Each stage has been discussed and summarized in this paper. It is noted that the performance of each stage of the emotion recognition system is interdependent. If the raw signal noise artifacts do not undergo preprocessing, the number of irrelevant features extracted can increase causing lengthy computational time. Besides that, various techniques and methods can be employed in the feature extraction (WT, FFT, HHT), feature selection (SFS, SBS, SFFS ) and classification (PNN, SVM). Selecting the most suitable techniques and methods for each stage is important as it will affect the recognition accuracy.

Emotion recognition from physiological signals still posses a number of challenges. Since physiological reactions are sensitive to motion artefacts, mapping the physiological patterns onto specific emotional states, especially in real-time, becomes increasingly difficult. Besides that, most of the current emotion recognition systems work with core emotions and smaller subsets of emotions such as depression, boredom and frustration. Fine-grained emotions such as hopelessness and hopefulness are rarely being studied as these emotions are hard to detect. Integrating other emotion recognition modalities such as face recognition, speech recognition or gesture recognition with physiological signals has shown promising results in recognizing emotional states [14]. However, there is a tradeoff between accuracy and computational time, which remains a worthy issues to be explored.

\section{REFERENCES}

[1] K. R. Scherer, "Appraisal theory. Handbook of cognition and emotion," in Handbook of cognition and emotion, T. Dalgleish and M. Power, Eds. John Wiley \& Sons Ltd, 1999, pp. 637-663.

[2] J.F.Cohn, "Foundations of human computing: Facial expression and emotion," In Proceedings of the International Conference on Multimodal Interfaces, pp. 233-238, 2006.

[3] Z.Zeng, M.Pantic, T.S.Huang, "Emotion Recognition Based on Multimodal Information," in Affective Information Processing; London: Springer Science \& Business Media LLC 2009, ch. 14, pp241 -265.

[4] J. Kim and E. André, "Emotion Recognition Based on Physiological Changes in Music Listening," IEEE Transactions on Pattern Analysis and Machine Intelligence, vol. 30, no. 12, pp. 2067-2083, 2008.

[5] D. Bradley, W. Heidrich, T. Popa, and A. Sheffer, "High resolution passive facial performance capture," ACM Transactions on Graphic., vol. 29, no. 4, pp. 41:1-41:10, Jul. 2010.

[6] J. Arroyo-Palacios and D. M. Romano, "Towards standardization in the use of Physiological Signals for Affective Recognition Systems," in Proceedings of the 6th International Conference of Methods and Techniques in Behavioral Research, pp. 121-124, 2008.

[7] P. Ekman, W. V Friesen, M. O'Sullivan, A. Chan, I. Diacoyanni-Tarlatzis, K. Heider, R. Krause, W. A. LeCompte, T. Pitcairn, and P. E. Ricci-Bitti, "Universals and cultural differences in the judgments of facial expressions of emotion.," Journal of Personality and Social Psychology, vol. 53, no. 4, pp. 712-717, 1987.

[8] P. J. Lang, "The Emotion Probe: Studies of Motivation and Attention," American Psychologist, vol. 50, pp. 372385, 1995.

[9] R. Plutchik, "Emotions and life: Perspectives from psychology, biology, and evolution," Psychology and Marketing, vol. 22, no. 1, pp. 97-101, 2002.

[10] A. Hanjalic and L.-Q. Xu, "Affective video content representation and modeling," IEEE Transactions on Multimedia, vol. 7, no. 1. pp. 143-154, 2005.

[11] S. Jerritta, M. Murugappan, R. Nagarajan, and K. Wan, "Physiological signals based human emotion Recognition: a review," in 2011 IEEE 7th International Colloquium on Signal Processing and its Applications, 2011, pp. 410-415.

[12] R.Henriques, A.Paiva, and C.Antunes, "On the need of new methods to mine electrodermal activity in emotioncentered studies, " in Lecture Notes in Computer Science, vol 7607,pp203 -215, 2013.

[13] S.D. Kreibig, "Autonomic nervous system activity in emotion: A review," Biological Psychology 84, 2010, pp394421.

[14] R. B. Knapp, J. Kim, and E. André, "Physiological Signals and Their Use in Augmenting Emotion Recognition for Human-Machine Interaction," in Emotion Oriented Systems, R. Cowie, C. Pelachaud, and P. Petta, Eds. Springer Berlin Heidelberg, 2011, pp. 133-159.

[15] Y. Cheng, G.-Y. Liu, and H. Zhang, The research of EMG signal in emotion recognition based on TS and SBS algorithm. 2010, pp. 363-366.

[16] M. Li, Q. Chai, T. Kaixiang, A. Wahab, and H. Abut, "EEG Emotion Recognition System," in In-Vehicle Corpus and Signal Processing for Driver Behavior SE, K. Takeda, H. Erdogan, J. L. Hansen, and H. Abut, Eds. Springer US, 2009, pp. 125-135.

[17] D. O. Bos, "EEG-based Emotion Recognition the Influence of Visual and Auditory Stimuli," Emotion, vol. 57, no. 7, pp. 1798-806, 2006.

[18] M. Bradley and P. Lang, "International affective digitized sounds (IADS): Stimuli, instruction manual and affective ratings," Gainesville, FL, 1999.

[19] P. Lang, M. Bradley, and B. N. Cuthbert, "International affective picture system (IAPS): Affective ratings of pictures and instruction manual," Gainesville, FL, 2008.

[20] J. J. Gross and R. W.Levenson, "Emotion Elicitation using Films,"Cognition and Emotion, vol. 9, pp. 87-108, 1995.

[21] J. T. Cacioppo, B. N. Uchino, S. L. Crites, M. A. Snydersmith, G. Smith, G. G. Berntson, and P. J. Lang, "Relationshipbetween facial expressiveness and sympathetic activation in emotion: a critical review, with emphasis on modeling underlying mechanisms and individual differences.," Journal of Personality and Social Psychology, vol. 62, no. 1, pp.110-128, 1992. 
[22] G. Rigas, C. D. Katsis, G. Ganiatsas, and D. I. Fotiadis, "A User Independent, Biosignal Based, Emotion Recognition Method," in User Modeling 2007, 2007, vol. 4511, pp. 314-318.

[23] B. B. D. Storey, "Computing Fourier Series and Power Spectrum with MATLAB," Time, vol. 93, no. 2, pp. 1-15, 2002. [24] K.Najarian and R.Splinter, Biomedical Signal and Image Processing, U.S:Taylor \& Francis Group, 2012, ch 4, pp79-99.

[25] S. Koelstra, C. Muhl, M. Soleymani, J.-S. Lee, A. Yazdani, T. Ebrahimi, T. Pun, A. Nijholt, and I. Patras, "DEAP: A Database for Emotion Analysis Using Physiological Signals," IEEE Transactions on Affective Computing, vol. 3, no. 1, pp. 18-31, 2012.

[26] C. Z. C. Zong and M. Chetouani, "Hilbert-Huang transform based physiological signals analysis for emotion recognition," IEEE 2009, pp. 334-339

[27] C. Maaoui, A. Pruski, and F. Abdat, "Emotion recognition for human-machine communication," IEEE/RSJ International Conference on Intelligent Robots and Systems, pp. 1210-1215, 2008

[28] E. M. Tamil, N. S. Bashar, M. Y. I. Idris, and A. M. Tamil, "A Review on Feature Extraction \& Classification Techniques for Biosignal Processing (Part III: Electromyogram)," in 4th Kuala Lumpur International Conference on Biomedical Engineering 2008, 2008, pp. 117-121.(cancel)

[29] A. K. Jain and P. W. Duin, "Statistical pattern recognition: a review," IEEE Transactions on Pattern Analysis and Machine Intelligence, vol. 22, no. 1, pp. 4-37, 2000.

[30] R. W. Picard, E. Vyzas, and J. Healey, "Toward machine emotional intelligence: Analysis of affective physiological state," vol. 23, no. 10. Published by the IEEE Computer Society, 2001, pp. 1175-1191.

[31] J. Arroyo-Palacios and D. M. Romano, "Bio-Affective Computer Interface for Game Interaction.," International Journal of Gaming and Computer Mediated Simulations, vol. 2, no. 4, pp. 16-32, 2010

[32] E.Jang, B.Park, S.Kim and J.Sohn, "Emotion Classification by Machine Learning Algorithm using Physiological Signals," in International Proceedings of Computer Science \& Information Technology, pp 1-5, vol 25, Singapore: IACSIT Press, 2012.

\section{Author's biography with Photo}

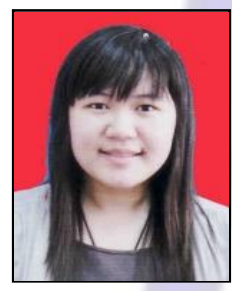

Heng Yu Ping is currently Ph.D candidate in Multimedia System at Universiti Putra Malaysia (UPM). She received her Master in Computer Science from UPM in 2010 and Bac. in Computer Science (Major In Multimedia) from UPM in 2008. Her research interests include facial modeling, facial animation, emotion recognition and physiological signals processing.

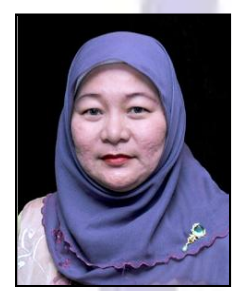

Lili Nurliyana Abdullah is an Associate Professor at Faculty of Computer Science and Information Technology(FCSIT), UPM. She received her Ph.D in Information Scence from Universiti Kebangsaan Malaysia in 2007, M.S degree in Engineering (Telematics) from University of Sheffield, United Kingdom in 1996, and Bac. in Computer Science from UPM in 1990. Her research interests include multimedia system, video processing and retrieval, computer modeling and animation, image processing and computer games.

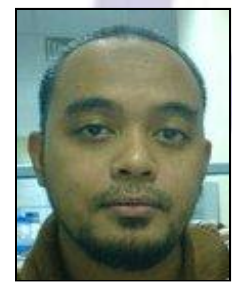

Alfian Abdul Halin is a senior lecturer at FCSIT, UPM. He obtained his Master of Multimedia Computing from Monash University in 2004, and the Ph.D in Computer Science from Universiti Sains Malaysia in 2011. His research interests include computer vision, image and audio processing, semantic concept detection, indexing and retrieval (images and videos) and applied machine learning.

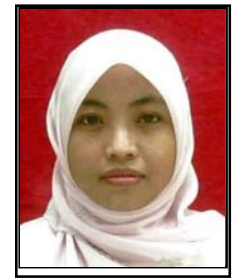

Puteri Suhaiza Sulaiman is currently a senior lecturer at FCSIT, UPM. She received her Ph.D in Computer Graphics from UPM in 2010, M.S. in Computer Science from Universiti Teknologi Malaysia (UTM) in 2003 and Bac. in Computer Science from UTM in 2000. Her research interests include computer graphics, geographical information systems, scientific visualization, medical visualization, flight simulation, virtual reality and computer games. 